\title{
Association Class
}

National Cancer Institute

\section{Source}

National Cancer Institute. Association Class. NCI Thesaurus. Code C45672.

In UML modeling, an association that is also a class. It not only connects a set of classes, but also defines a set of features that belong to the relationship itself. 